\title{
A Klebsiella pneumoniae ST307 outbreak clone from Germany demonstrates features of extensive drug resistance, hypermucoviscosity, and enhanced iron acquisition
}

Stefan E. Heiden ${ }^{1 \dagger}$, Nils-Olaf Hübner ${ }^{2+}$, Jürgen A. Bohnert ${ }^{3}$, Claus-Dieter Heidecke ${ }^{4}$, Axel Kramer ${ }^{5}$, Veronika Balau ${ }^{6}$, Wolfgang Gierer ${ }^{7}$, Stephan Schaefer ${ }^{7}$, Tim Eckmanns ${ }^{8}$, Sören Gatermann ${ }^{9}$, Elias Eger ${ }^{1}$, Sebastian Guenther ${ }^{10}$, Karsten Becker $^{3}$ and Katharina Schaufler ${ }^{1 *}$ (D)

\begin{abstract}
Background: Antibiotic-resistant Klebsiella pneumoniae are a major cause of hospital- and community-acquired infections, including sepsis, liver abscess, and pneumonia, driven mainly by the emergence of successful high-risk clonal lineages. The $K$. pneumoniae sequence type (ST) 307 lineage has appeared in several different parts of the world after first being described in Europe in 2008. From June to October 2019, we recorded an outbreak of an extensively drug-resistant ST307 lineage in four medical facilities in north-eastern Germany.
\end{abstract}

Methods: Here, we investigated these isolates and those from subsequent cases in the same facilities. We performed whole-genome sequencing to study phylogenetics, microevolution, and plasmid transmission, as well as phenotypic experiments including growth curves, hypermucoviscosity, siderophore secretion, biofilm formation, desiccation resilience, serum survival, and heavy metal resistance for an in-depth characterization of this outbreak clone.

Results: Phylogenetics suggest a homogenous phylogram with several sub-clades containing either isolates from only one patient or isolates originating from different patients, suggesting inter-patient transmission. We identified three large resistance plasmids, carrying either NDM-1, CTX-M-15, or OXA-48, which K. pneumoniae ST307 likely donated to other K. pneumoniae isolates of different STs and even other bacterial species (e.g., Enterobacter cloacae) within the clinical settings. Several chromosomally and plasmid-encoded, hypervirulenceassociated virulence factors (e.g., yersiniabactin, metabolite transporter, aerobactin, and heavy metal resistance genes) were identified in addition. While growth, biofilm formation, desiccation resilience, serum survival, and heavy metal resistance were comparable to several control strains, results from siderophore secretion and (Continued on next page)

\footnotetext{
* Correspondence: katharina.schaufler@uni-greifswald.de

${ }^{\dagger}$ Stefan E. Heiden and Nils-Olaf Hübner shared the first authorship.

'Institute of Pharmacy, Pharmaceutical Microbiology, University of Greifswald,

Friedrich-Ludwig-Jahn-Str. 17, 17489 Greifswald, Germany

Full list of author information is available at the end of the article
}

(c) The Author(s). 2020 Open Access This article is licensed under a Creative Commons Attribution 4.0 International License, which permits use, sharing, adaptation, distribution and reproduction in any medium or format, as long as you give appropriate credit to the original author(s) and the source, provide a link to the Creative Commons licence, and indicate if changes were made. The images or other third party material in this article are included in the article's Creative Commons licence, unless indicated otherwise in a credit line to the material. If material is not included in the article's Creative Commons licence and your intended use is not permitted by statutory regulation or exceeds the permitted use, you will need to obtain permission directly from the copyright holder. To view a copy of this licence, visit http://creativecommons.org/licenses/by/4.0/. The Creative Commons Public Domain Dedication waiver (http://creativecommons.org/publicdomain/zero/1.0/) applies to the data made available in this article, unless otherwise stated in a credit line to the data. 


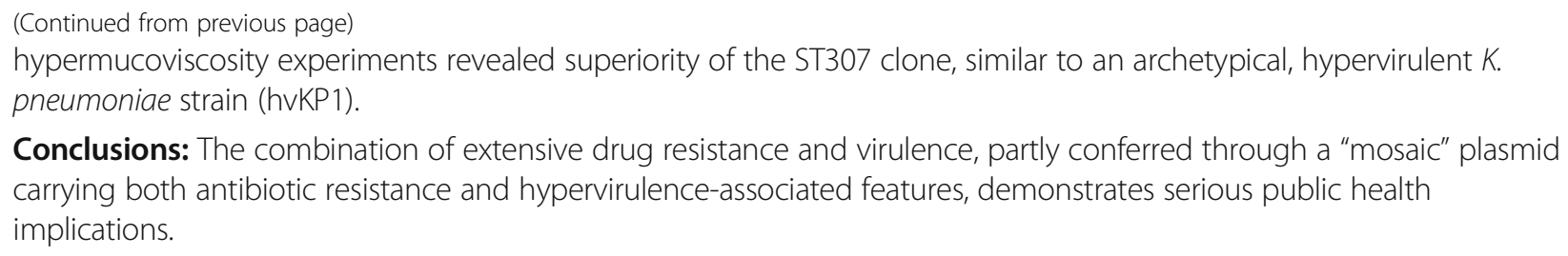

Keywords: XDR Klebsiella pneumoniae, Outbreak, Hypervirulence, Plasmid transmission, "Mosaic" plasmid

\section{Background}

Klebsiella pneumoniae cause severe infections including sepsis, liver abscess, and pneumonia [1, 2]. The emergence of multidrug-resistant (MDR) and extensively drug-resistant (XDR), "classic" K. pneumoniae (cKp) has been mainly driven by the dissemination of high-risk clonal lineages and now constitutes a major global public health problem [3]. The majority of cKp strains cause infection in immunocompromised patients in healthcare settings and have demonstrated the ability to acquire antibiotic resistance elements. In addition to this cKp, a second pathotype termed hypervirulent $K$. pneumoniae (hvKp) is currently circulating, particularly in Asia but with increasing reports from other countries [2, 4]. The hvKp's defining features are clinical characteristics including multiple site infection and metastatic spread in the healthy community [5] and, originally, a positive laboratory string test indicating a hypermucoid phenotype [6]. The definition of hypervirulence is controversial, however [7]. A recent study identified potential biomarkers including peg-344, iroB, iucA, plasmid-borne rmpA and rmpA2 genes, and high siderophore production in hvKp to accurately differentiate the two pathotypes [8].

While K. pneumoniae sequence type (ST) 258 has been recognized as antibiotic-resistant, high-risk clonal lineage [9], ST307 came into spotlight only more recently $[3,10,11]$. The first $K$. pneumoniae ST307 isolates were obtained in the Netherlands in 2008 and Pakistan in 2009, followed by a period of sporadic isolations across Europe, Asia, Africa, and the Americas [11], and originated mostly, but not uniquely, from clinical samples $[3,10]$. A phylogenetic study suggests the emergence of two deep-branching ST307 lineages, one of global relevance with genomes from locations worldwide and evidence of intercontinental transfer [10]. The other lineage included $K$. pneumoniae ST307 isolates from Texas, USA [12]. Within the global lineage, some isolates also originated from the infections in Texas [12], which suggests that the USA may have been the origin of this sequence type, especially as most of its genetic diversity was present in that location [10]. ST307 often carries transferable resistance-conferring genes against carbapenems and newer-generation cephalosporins like $b l a_{\mathrm{KPC}-3}, b l a_{\mathrm{NDM}-1}, b l a_{\mathrm{OXA}-48}$, and $b l a_{\mathrm{CTX}-\mathrm{M}-15}[3,10]$. Resistance to the novel combination ceftazidime/avibactam [13] and to colistin was also reported [14, 15]. Besides that, the ST307 K. pneumoniae lineage comprises a variety of additional resistance and virulence determinants, integrative conjugative elements, and phages $[3,10]$. There are several outbreak reports on MDR $K$. pneumoniae ST307 in clinical settings [16-18].

In this study, we analyzed carbapenemase-producing ST307 isolates, which have been recovered from screening and clinical samples within the course of an outbreak [19] that took place in four medical facilities in Western Pomerania, Germany, from June 2019 to October 2019. Additional cases were detected after the actual outbreak in the beginning of 2020. These isolates were characterized as carrying NDM-1 and OXA-48 carbapenemaseencoding genes, mostly simultaneously, and tested colistin-resistant. We performed whole-genome sequencing and phenotypic experiments to enable functional genomics for the in-depth understanding of this XDR outbreak clonal lineage.

\section{Methods}

\section{Sequenced isolates and metadata}

Between June 2019 and February 2020, we investigated 56 enterobacterial isolates from 25 different patients involved in the outbreak. In addition to K. pneumoniae, we included all other Enterobacteriaceae that matched the carbapenem-resistant phenotype. Most isolates were obtained from rectal $(n=23)$ or throat/tracheal secretion $(n=17)$ swabs collected as part of an extensive surveillance program in the affected clinical institutions (Additional file 2: Table S1). Initial antibiotic susceptibility testing (AST) was performed using the VITEK 2 (bioMérieux, Nürtingen, Germany) system and 96-well plate broth microdilution (Merlin, Bornheim-Hersel, Germany). Bacterial species were initially identified by MALDI-TOF MS (VITEK MS, bioMérieux, Nürtingen, Germany). For rapid detection of carbapenemase genes, a loop-mediated isothermal amplification (LAMP) assay (eazyplex SuperBug CRE, AmplexDiagnostics, Gars, Germany) was subsequently included in the University Medicine laboratory diagnostic program [19]. Almost $59 \%(33 / 56)$ of the isolates were assigned to "infection" 
samples. Clinical data and outcomes of 17 initial cases have been published elsewhere [19]. Most of these had severe underlying diseases. Patients were treated with ceftazidime-avibactam/aztreonam with synergistic activity. By October, six patients had died. Causal associations with the outbreak clone as well as clinical outcomes of other cases are still under investigation, however. Epidemiologic links among the different institutions were identified (Additional file 2: Table S1). Samples were incubated overnight on CHROMID CARBA and CHROMID ESBL agar plates (bioMérieux, Nürtingen, Germany), and single colonies were picked for identification with VITEK MS (bioMérieux, Nürtingen, Germany). Antimicrobial susceptibility testing was carried out using VITEK 2 (bioMérieux, Nürtingen, Germany), and in addition, 96-well plate broth microdilution was performed for determination of colistin MICs (Merlin, Bornheim-Hersel, Germany).

\section{Whole-genome sequencing}

We generated $52 \mathrm{~K}$. pneumoniae, $2 \mathrm{E}$. coli, $1 \mathrm{C}$. freundii, and $1 E$. cloacae whole-genome sequences on different sequencing machines (MiGS: Illumina NextSeq 550; LGC: Illumina NextSeq 500; Eurofins: Illumina NovaSeq 6000). Two K. pneumoniae isolates (PBIO1953 [va20750], PBIO1951 [va19352]) were long-read sequenced using ONT's Nanopore system. Additional file 2: Table S1 shows the respective metadata including origin institution, sampling location, and patient pseudonym. From 13 patients, more than one isolate was sequenced (11 patients with more than one K. pneumoniae isolate; 9 patients with more than one K. pneumoniae ST307 isolate). Some ST307 isolates were obtained from multiple sites from the same patient (e.g., PT17). DNA was extracted using the MasterPure $^{\mathrm{Tm}}$ DNA Purification Kit for Blood, Version II (Lucigen, Middleton, USA). After quantification and initial quality control, DNA was shipped to MiGS (Microbial Genome Sequencing Center, Pittsburgh, USA), LGC (LGC Genomics GmbH, Berlin, Germany), and Eurofins (Eurofins Genomics Europe Sequencing GmbH, Constance, Germany) and following library preparation with [20] (MiGS), the SeqWell ${ }^{\mathrm{TM}}$ Kit (LGC), and an adapted

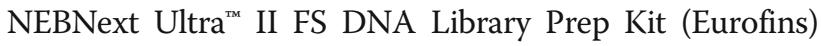
sequenced using $2 \times 150$ bp paired-end reads.

\section{Assembly and annotation}

Raw sequencing reads were adapter-trimmed, contaminantfiltered, and quality-trimmed using BBDuk from BBTools v. 38.41 (http://sourceforge.net/projects/bbmap/). Both trimmed reads and raw reads were quality-controlled using FastQC v. 0.11.8 (http://www.bioinformatics.babraham.ac.uk/projects/ fastqc/). De novo genome assemblies were conducted by employing the assembly pipeline shovill v. 1.0.4 (https:// github.com/tseemann/shovill) in combination with SPAdes v.
3.13.1/3.14.0 [21]. As part of the pipeline, trimmed reads were subsampled to assemble at a maximum coverage of $100 \times$. Besides the polishing step as part of the shovill pipeline, assemblies underwent an additional polishing step. For this, all trimmed reads were mapped back to the contigs using bwa v. 0.7.17 [22]. The obtained SAM/BAM files were sorted with Samtools v. 1.9 [23] and optical duplicates marked with GATK v. 4.1.2.0 [24]. Finally, variants were called with Pilon v. 1.23 [25]. The genomes of strains for which additional long-read sequencing data were obtained were hybridassembled with Unicycler v. 0.4.8 [26]. To verify the "hybrid" nature of plasmid 1, we mapped the long-reads of PBIO1953 back to the assembly using minimap2 v. 2.17 [27] and visualized the alignment with Tablet v. 1.19.09.03 [28] (Additional file 1: Fig. S1). The assembly graphs of putative plasmid recipient isolates and the closed reference isolate PBIO1953 were inspected with Bandage v. 0.8.1 [29] and its integrated BLAST hit (Megablast, $\geq 99 \%$ identity, $E$ value $1 \mathrm{e}$ -10) visualization (Additional file 1: Fig. S2). Genome quality and completeness were assessed with CheckM v. 1.0.13 [30]. We used Prokka v. 1.14.1 [31] to annotate draft and finished genomes automatically.

\section{Genomic analysis}

The in silico multi-locus sequence typing (MLST) and antibiotic resistance/virulence gene detection were carried out using mlst v. 2.19.0 (https:/github.com/tseemann/mlst) and ABRicate v. 0.9.9 (https:/github.com/ tseemann/abricate), respectively. Both tools rely on 3rdparty public databases (e.g., PubMLST [32], VFDB [33], ResFinder [34], PlasmidFinder [35], BacMet [36]). To visualize draft genome content, BRIG v. 0.95-dev.0004 [37] and NCBI BLAST v. 2.9.0+ [38] with a threshold of at least $99 \%$ identity were employed by aligning isolate contigs against the respective reference. Pangenome analysis was performed with Roary v. 3.12.0 [39]. For indepth typing of yersiniabactin, aerobactin, $\mathrm{K}$ locus, and O locus, we used Kleborate v. 1.0.0 with Kaptive [4043]. A synteny plot comparing plasmid 1 (pPBIO1953 NDM-1) and plasmid 2 (pPBIO1953_CTX-M-15) of PBIO1953 with $K$. pneumoniae virulence plasmids pK2044 [44] and pKCTC2242 [45] was created with genoPlotR v. 0.8.9 [46].

\section{Phylogeny}

We created a core SNP phylogeny for ST307 including (a) only the 44 ST307 isolates from this study (outbreak phylogeny) (Fig. 1) and (b) the 44 ST307 isolates as well as 97 publicly available ST307 genomes (79 as raw reads, 18 as assembly) (global phylogeny) (Additional file 2: Table S2). SNPs were called from the finished genome of PBIO1953 using snippy v. 4.4.1 (https://github.com/ tseemann/snippy). Alignments were filtered for recombinations using Gubbins v. 2.4.1 [47] and core SNPs 


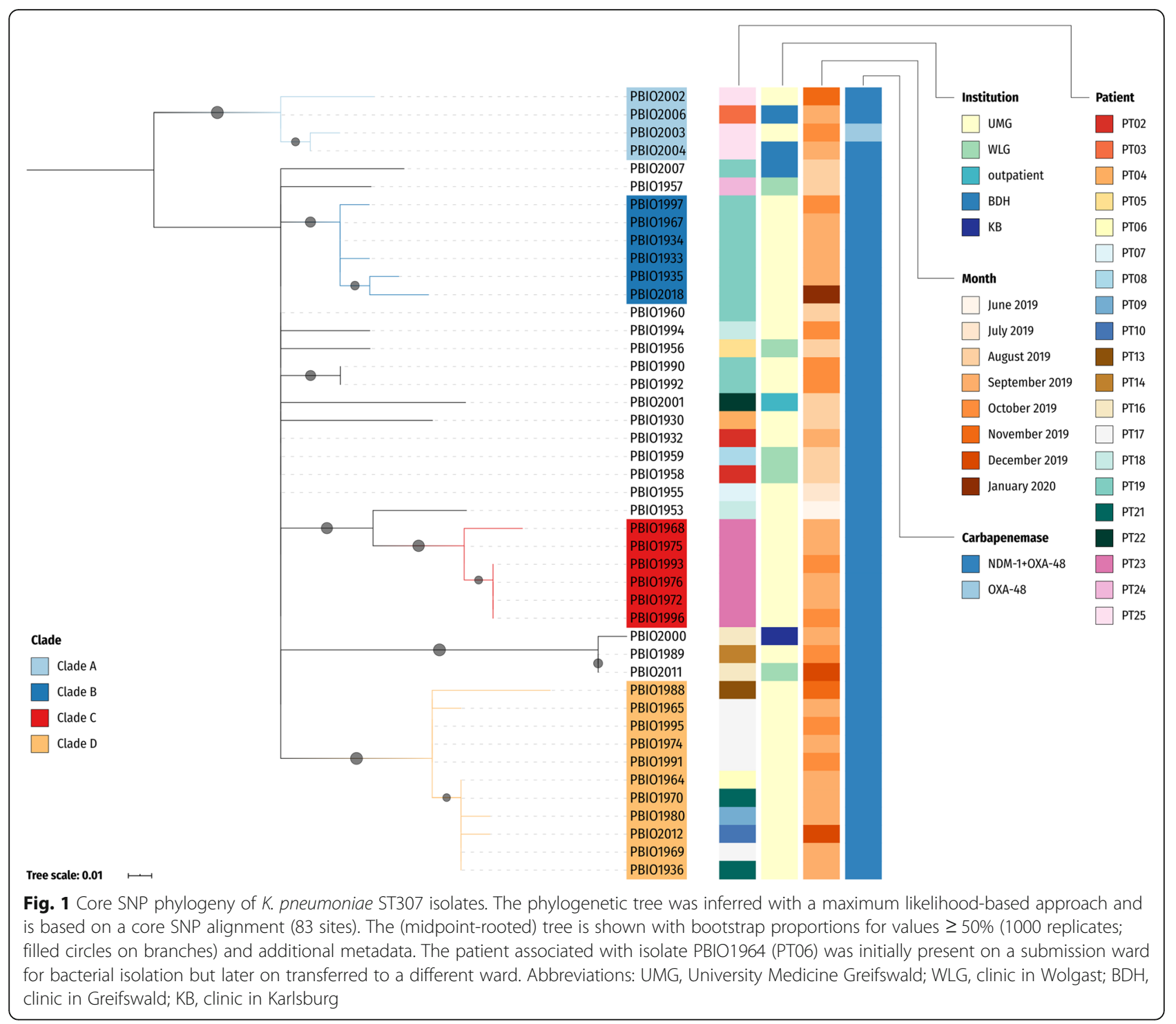

extracted using snp-sites v. 2.5.1 (83 sites for the outbreak phylogeny; 1476 sites for the global phylogeny) [48]. A maximum likelihood tree was inferred with RAxML-NG v. 0.9.0 [49] using GTR+G. The bestscoring maximum likelihood tree was midpoint-rooted in iTOL v. 5.5 [50] and visualized with metadata. An additional phylogenetic tree of all isolates was inferred based on whole genomes with the help of JolyTree v. 1.1b.191021ac [51] and visualized with iTOL v. 5.5 [50].

\section{Pathway analysis of variants}

Single-nucleotide polymorphisms detected among the $K$. pneumoniae ST307 genomes were filtered for missense, frameshift, and stop-gained variants and respective genes subjected to metabolic pathway analysis using the EcoCyc (https://ecocyc.org/) [52] database (reference: Klebsiella pneumoniae "KpvST383_NDM_OXA-48" [NCBI Biosample number: SAMN10409888] [53]).

\section{Bacterial isolates and PCV construction}

For phenotypic experiments, we used a selection of five $K$. pneumoniae ST307 outbreak isolates (PBIO1953, PBIO2000, PBIO1935, PBIO1994, PBIO1993) and three non-ST307 K. pneumoniae present during the outbreak (PBIO1951 and PBIO1961 [ST395], PBIO1979 [ST405]) (Additional file 2: Table S1). They were compared against three $K$. pneumoniae with different sequence types: a common ESBL-producing reference (ATCC700603 [ST498]), one of the ubiquitously occurring K. pneumoniae lineage ST15 (PBIO2008), and one archetypal, hypervirulent $K$. pneumoniae strain (hvKP1, ST86) $[6,54]$. In addition, we included a non-carbapenemase-producing $K$. pneumoniae ST307 isolate from a previous study 
(IMT38405 [PBIO2009]) [11] and a "plasmid-cured" variant (PCV1935) stemming from PBIO1935. Based on a previously published protocol [55], this mutant was constructed by culturing PBIO1935 for 7 days at $42^{\circ} \mathrm{C}$ and using ceftazidime/avibactam $(16 \mu \mathrm{g} / \mathrm{mL})$ as selection marker to identify NDM-1-plasmid-cured variants. Plasmid profile analysis (Additional file 1: Fig. S3A) performed as previously described [55], and bioinformatics analysis confirmed partial loss of plasmid 1 (Additional file 1: Fig. S3A; dashed box) and complete loss of plasmid 3 (Additional file 1: Fig. S3A; dotted box) in PCV1935.

\section{Growth curves}

Growth curves in LB medium were performed using standardized protocols. Experiments were performed using three technical replicates and three biological replicates [56]. An E. coli K-12 strain (W3110) was used as control. Growth rates were calculated as follows: $\mu=$ $\left(\ln \left(\mathrm{CFU} / \mathrm{mL} t_{1}\right)-\ln \left(\mathrm{CFU} / \mathrm{mL} t_{0}\right)\right) / t_{1}-t_{0}$.

\section{Hypermucoviscosity}

Hypermucoviscosity experiments were performed using the string test. Strings of $5 \mathrm{~mm}$ or longer that formed after stretching on the tip of a sterile inoculation loop were defined positive [6]. Experiments were performed with three technical replicates and three biological replicates.

\section{Siderophore secretion}

We analyzed the study's set of isolates for their ability to secrete siderophores using an adapted method described by Schwyn and Neilands [57]. Fifty microliters of overnight cultures of the isolates was grown in $5 \mathrm{~mL}$ fresh $\mathrm{LB}$ medium to an $\mathrm{OD}_{600}$ of 0.6. Five microliters of this culture was put on agar plates containing chrome azurol S-iron(III)-hexadecyltrimethylammonium bromide and incubated overnight at $37^{\circ} \mathrm{C}$. Iron uptake was determined visually by color shift from blue to yellow the following day. One ST131 E. coli strain (IMT21183) and one K-12 E. coli strain (W3110) were included as controls [58]. Experiments were performed with three technical replicates and three biological replicates.

\section{Biofilm formation}

We used the same set of isolates as described above and performed a biofilm formation experiment as described in previous reports [59]. Ten microliters of overnight cultures of all isolates was transferred to $1 \mathrm{~mL} \mathrm{LB}$ medium and cultured at $37^{\circ} \mathrm{C}$ until $\mathrm{OD}_{600}$ values of $0.6-$ 0.8 were reached. One hundred microliters of culture per well was transferred to a 96 -well microtiter plate. After 3 days of static culture at $37^{\circ} \mathrm{C}$, planktonic cells were removed from the liquid medium. The wells were washed three times with $150 \mu \mathrm{L}$ of double-distilled water, and the majority of the biofilms was stained with $150 \mu \mathrm{L}$ of $0.1 \%$ crystal violet $(\mathrm{CV})$ for $30 \mathrm{~min}$. Then, the unbound dye was removed, and the plates were again washed. Finally, the CV binding to the biofilm was dissolved in $150 \mu \mathrm{L}$ of $95 \%$ ethanol for $30 \mathrm{~min}$, and biofilm formation was quantified by measuring the absorbance at $\mathrm{OD}_{590}$ with a microplate reader (Fluostar Omega, BMG Labtech, Ortenberg, Germany). Experiments were performed with three technical replicates on individual 96-well microtiter plates and three biological replicates. One biofilm-negative (PBIO729) and one positive control (W3110) [56] were included.

\section{Serum resistance}

We performed serum resistance experiments in human serum (Pan-Biotech $\mathrm{GmbH}$, Germany) as described previously [60]. We inoculated $5 \mu \mathrm{L}$ of overnight culture in $495 \mu \mathrm{L}$ fresh LB medium and incubated at $37^{\circ} \mathrm{C}$ for 1.5 h. Inoculum was centrifuged for $3 \mathrm{~min}$ and resuspended in $1 \mathrm{~mL}$ of sterile $1 \times$ PBS. Thirty microliters was added in triplicates to 96-well microtiter plates containing $270 \mu \mathrm{L}$ of $50 \%$ human serum. Thirty microliters of the sample was collected from each well, serially diluted, plated on LB plates, grown at $37^{\circ} \mathrm{C}$ overnight, and counted the next day (0-h count). The 96 -well microtiter plates were incubated for $4 \mathrm{~h}$ at $37^{\circ} \mathrm{C}$. Following incubation, $30 \mu \mathrm{L}$ of the culture was plated, incubated, and again counted (4-h count). Growth in serum was obtained by determining differences in the CFU after $4 \mathrm{~h}$ of incubation compared to the 0 -h count. Experiments were performed with three technical replicates on individual 96-well plates and three biological replicates. An E. coli serum-resistant control (PBIO1289, ST1159 [61]) was included.

\section{Desiccation tolerance}

Desiccation tolerance experiments for the study's set of isolates were performed as described previously with some modifications [59]. A single colony was cultured in $10 \mathrm{~mL}$ liquid LB broth until bacterial cells reached an $\mathrm{OD}_{600}$ value of $0.6-0.8$. One hundred microliters was serially diluted, plated on LB plates, grown at $37^{\circ} \mathrm{C}$ overnight, and counted the next day (COd, 0-day count). Another $100 \mu \mathrm{L}$ of the same culture was transferred into a 96-well microtiter plate. Subsequently, the plate was transferred into a sterile dryer with dehydrated silica gel. The dryer was placed in a sterile incubator (Mytron, Heilbad Heiligenstadt, Germany), which was kept at a constant temperature of $37^{\circ} \mathrm{C}$. After 6 days of drying, the 96 -well microtiter plate was removed, $100 \mu \mathrm{L} /$ well of fresh medium was added, and the plate was cultured with $200 \mathrm{rpm}$ shaking at $37^{\circ} \mathrm{C}$ for $3 \mathrm{~h}$. One hundred microliters was collected from each well, serially diluted, plated on LB plates, grown at $37{ }^{\circ} \mathrm{C}$ overnight, and 
counted the next day (C6d, 6-day count). Experiments were performed with three technical replicates on individual 96-well microtiter plates and three biological replicates. An E. coli K-12 strain (W3110) was included as control.

\section{Heavy metal tolerance}

Overnight cultures of the study's set of isolates were adjusted to McFarland standard 0.5, and $50 \mu \mathrm{L}$ of a 1:200 dilution of adjusted suspensions in Mueller-Hinton broth (Roth, Karlsruhe, Germany) was used as inoculum for incubations for 16 to $20 \mathrm{~h}$ at $37^{\circ} \mathrm{C}$ in heavy metalcontaining microtiter plates (Merlin Biocide plates, Bornheim-Hersel, Germany). The plates contained a wide range of concentrations of three heavy metals: zinc chloride [4-8192 $\mu \mathrm{g} / \mathrm{mL}$ ], copper sulfate [32-8192 $\mu \mathrm{g} /$ $\mathrm{mL}]$, and silver nitrate $[0.5-64 \mu \mathrm{g} / \mathrm{mL}]$. We used a sealing tape to prevent dehydration of the plates. After incubation, the minimum inhibitory concentration was determined visually and reported as the tolerance breakpoint. Experiments were performed with three technical and three biological replicates. E. coli ATCC25922 was used as control isolate.

\section{Statistics}

Statistics were performed using GraphPad Prism 8.0 (https://www.graphpad.com/). After investigating Gaussian distributions, the non-parametric Kruskal-Wallis test [62] was applied for multiple comparisons of bacterial groups using median values. Bonferroni adjustment was applied, which resulted in corrected $p$ values of $p<0.016$ to assess significant changes [63]. Pairwise comparison of growth rates $(\mu)$ between all $K$. pneumoniae ST307 isolates and PBIO1961 was performed using the MannWhitney $U$ test $(p<0.05)$.

\section{Results}

\section{Genomic analysis and phylogeny}

The XDR K. pneumoniae outbreak clone was first detected at the University Medicine Greifswald on June 25, 2019 (Additional file 2: Table S1), following bacterial screening of a tracheal secretion sample [19]. Of $52 \mathrm{~K}$. pneumoniae, 44 belonged to sequence type (ST) 307, three to ST395, three to ST11, and one each to ST405 and ST147. The two E. coli were ST405 and ST362, whereas C. freundii was a ST153 and E. cloacae a ST45 isolate (Additional file 2: Table S1). We focused mainly on the phylogenetics and phenotypes of the ST307 outbreak but included accessory and non-ST307 genomes to investigate transmission of resistance plasmids within the bacterial species and to others.

When comparing all 44 ST307 against the closed reference genome of PBIO1953, our analysis revealed 22 singlenucleotide polymorphisms (SNPs) at most (minimum, 6
[PBIO1958, PBIO1960, PBIO1932]; maximum, 22 [PBIO2004]; median, 11). As expected, the phylogram shows a homogenous picture (Fig. 1; Additional file 1: Figure S4) with several sub-clades. There are sub-clades that include only isolates originating from the same patient (clades B [811 SNPs compared to PBIO1953] and C [6-8 SNPs compared to PBIO1953]) and sub-clades that comprise isolates from different patients (clades A [14-17 SNPs compared to PBIO1953] and D [11-15 SNPs compared to PBIO1953]) suggesting recent transmission between patients. Epidemiologic data support these results, for example for sub-clade D: PT17 with isolates PBIO1965, PBIO1969, PBIO1974, PBIO1991, PBIO1995, and PT21, from whom we obtained isolates PBIO1936 and PBIO1970, stayed on the same ward during the same time (September 2019). On the contrary, PT06 with isolate number PBIO1964 was present on a different ward during September 2019. Note, however, that PT06 was transferred later on. Interestingly, this patient underwent endoscopy examination with the same endoscope used for PT17 and PT21. It thus seems possible that $K$. pneumoniae ST307 was transmitted among patients either by crosscontamination through healthcare workers and surfaces or by an endoscope as has been described previously [64].

When we placed the outbreak phylogeny in a global context (Additional file 1: Fig. S4), we noticed that a cluster of KPC-producing ST307 genomes originally obtained from the United Kingdom (UK) (Additional file 2: Table S2) was the phylogenetically closest to our ST307 outbreak isolates.

We then explored the distribution and character of SNPs among the ST307 genomes (with PBIO1953 as reference) further (Additional file 1: Fig. S5). All polymorphisms that were not in coding sequences (CDS) or not assigned as missense, frameshift, or stopgained variants were excluded from our subsequent analysis. We further excluded all insertion and/or deletion mutations (Indels). Variants accumulated almost uniquely in chromosomally encoded genes (59/ 66). When analyzing the data of 44 annotated genes after exclusion of hypothetical proteins in EcoCyc, we noticed that pathways related to membrane transport (14/44), regulation and signal transduction (9/44), amino acid and sugar metabolism (12/44), DNAreplication/conjugation (5/44), and lipopolysaccharide biosynthesis $(4 / 44)$ were often affected by mutations. Thirty-seven genomes harbored a missense SNP in the conjugation gene traI (plasmid 3) and, simultaneously, the topoisomerase gene gyrA, which are both involved in plasmid conjugation and transfer $[65,66]$. Interestingly, all potential K. pneumoniae ST307 plasmid donors belonged to this set of genomes.

We often found variants in genes encoding for membrane efflux. While in sub-clade $C$, sotB was affected, in other clusters, we observed missense mutations in $p h o E$, 
$g l t C$, and $\operatorname{omp} C$. This might be an example for phenocopy in isolates of different sub-clades. The nitrate/nitrite sensor gene narX, differentiating sub-clades B (PT19) and D (various patients), and sensor protein pmrB, differentiating sub-clade C (PT23) from other sub-clades (various patients), were repeatedly and independently mutated among different patients. In addition, two other genes, narI and narJ, displayed SNPs; both are involved in the regulation of anaerobic respiratory gene expression in response to nitrate and nitrite.

Several genomes obtained from the same patient demonstrated identical variants not present in other genomes, which could be explained by either a disruption of the infection chain between patients or the non-advantageous character of the mutation for dissemination. One example is the variant in $b t s T$, a gene involved in pyruvate uptake and present in two isolates from the same patient over a period of 7 days. Interestingly, the number of missense/nonsense SNPs did not significantly increase over time during the course of the outbreak compared to the earliest isolate PBIO1953 (for example PBIO1955, 8 SNPs; PBIO1956, 11 SNPs; PBIO1957, 11 SNPs; PBIO2011, 11 SNPs; PBIO2012, 13 SNPs; and PBIO2018, 12 SNPs; Additional file 1: Fig. S5, top).

All ST307, with the exception of PBIO2003, carried $b l a_{\mathrm{NDM}-1}, b l a_{\mathrm{OXA}-48}$, and $b l a_{\mathrm{CTX}-\mathrm{M}-15}$ resistance genes simultaneously, which was consistent with their phenotypes. Due to the fact that $m c r$-genes were not present, phenotypic resistance against colistin could not be explained by the expression of such. We identified several missense mutations in the two-component systems PhoP/PhoQ (phoQ: $89 \mathrm{~T}>\mathrm{A}$ [Leu30Gln]) and PmrA/PmrB (pmrA: 121G $>$ A [Ala41Thr]; pmrB: 637C >A [Leu213Met], 766G>C [Gly256Arg]), typically involved in colistin resistance conferred by chromosomal point mutations [67]. Additional mutations were present in eptA $\quad(p m r C) \quad(80 \mathrm{~T}>\mathrm{G} \quad[\mathrm{Phe} 27 \mathrm{Cys}]), \quad p m r D \quad(179 \mathrm{C}>\mathrm{T}$ [Thr60Met]), arnT (1115A $>$ G [Lys372Arg], 1211C $>\mathrm{G}$ [Ser404Cys]), and ugd (1061A $>C$ [Asp354Ala]) (Additional file 1: Fig. S6). Three mutations (pmrB: 766G>C; eptA: 80T>G; arnT: 1115A $>$ G) were present in colistin-susceptible isolate PBIO1979 (ST405). Among the phenotypically colistin-resistant isolates, four carried additional mutations in pmrA (PBIO2001: $518 \mathrm{~T}>\mathrm{A} \quad$ [lle173Asn], $533 \mathrm{~T}>\mathrm{C}$ [Ile178Thr]), pmrB (PBIO1953: 364G>C [Glu122Gln]), or phoP (PBIO1990, PBIO1992: 563A $>C$ [His188Pro]). Interestingly, one missense mutation in $p m r B(604 \mathrm{C}>\mathrm{A}$ [Gln202Lys]) was exclusively present in isolates from patient PT23. While the amino acid substitutions in PmrA/PmrB (Ala41Thr/Leu213Met; Gly256Arg), together with an insertional inactivation of $m g r B$, were previously reported in a colistin-resistant K. pneumoniae ST307 isolate in 2015 [14], both this study's colistin-susceptible and colistinresistant isolates showed an uninterrupted $\operatorname{mgrB}$, whose gene product is a small negative regulator of PhoQ. The colistin-resistance phenotype is possibly explained by the combination of several mutations in chromosomally encoded genes.

Our analysis revealed that all ST307 genomes were positive for the chromosomally encoded "yersiniabactin $(y b t)$ lineage 10" genetic makeup, associated with the $K$. pneumoniae integrative conjugative element 4 (ICEKp4). The corresponding yersiniabactin sequence type (YbST) was $20-2 \mathrm{LV}$, which is most similar to YbST20 but varies in two loci (irp2: allele 61 instead of allele 208; fyuA: allele 39 instead of allele 2). All ST307 shared "aerobactin lineage iuc 1" signatures with aerobactin sequence type (AbST) 63-1LV and 63 (PBIO2003); thus, all ST307 isolates but one had a single-locus variant of AbST63 (SNP in iutA). Capsule (K) and $\mathrm{O}$ antigen loci of $\mathrm{K}$. pneumoniae ST307 isolates were predicted as KL102 and O2 variant $2(\mathrm{O} 2 \mathrm{v} 2)$ with identities of $\geq 99.25 \%$ and $\geq$ $98.45 \%$, respectively (Additional file 2 : Table S1).

Interestingly, the NDM-1, CTX-M-15, and OXA-48 resistances were located on three different, large plasmids, based on their respective size subsequently termed plasmids 1 (pPBIO1953_NDM-1), 2 (pPBIO1953_CTXM-15), and 4 (pPBIO1953_OXA-48) (Additional file 1: Fig. S3 and S7). Plasmid 1 (size $=360,596$ bp; incompatibility [Inc] types: IncFIB, IncHI1B) did not only encode the New-Delhi metallo-beta-lactamase (NDM)-1 but also several other resistance genes (dfrA5, sul1, sul2, qurS1, aph (3')-Ia, aph (3')-VI, $\operatorname{armA}, \operatorname{mph}(\mathrm{A}), \operatorname{mph}(\mathrm{E})$, and $m s r(\mathrm{E}))$ as well as virulence (peg-344/pagO, peg-1860/ pagO [metabolite transporter], iucABCD, iutA [aerobactin], and rmpA/rmpA2 [regulator of mucoid phenotype]) and disinfectant/mineral resistance factors (qacEdelta1 [disinfectant resistance], ter [tellurite resistance]) (Additional file 1: Fig. S7B). Plasmid 2 (size $=130,131 \mathrm{bp}$; IncFIB) carried the following antibiotic resistances: $b l a_{\mathrm{CTX}-\mathrm{M}-15}, b l a_{\mathrm{TEM}-1 \mathrm{~B}}$, sul2, aac (3)-IIa, aph (3")-Ib, and aph (6)-Id, and, interestingly, several genes responsible for heavy metal resistance: ars (arsenic/antimony resistance), sil (silver resistance), and pco (copper resistance). We compared plasmids 1 and 2 to two wellcharacterized, typical virulence plasmids of hypervirulent K. pneumoniae NTUH-K2044 [44] and KCTC 2242 [45]. We found a high degree of similarity in the aforementioned virulence and mineral resistance features (Fig. 2). This is of particular public health interest as the emergence of "mosaic" plasmids carrying AMR and virulence factors can confer both enhanced virulence and multidrug resistance in one single transfer. Plasmid 4 (size $=$ $63,589 \mathrm{bp}$; IncL/M) was positive for the OXA-48encoding gene. Two additional plasmids with sizes of 72, $679 \mathrm{bp}$ (plasmid 3; IncFII) and $6656 \mathrm{bp}$ (plasmid 5; Col440I) did not carry any resistance or virulence genes (Additional file 1: Fig. S7D and S7F). 


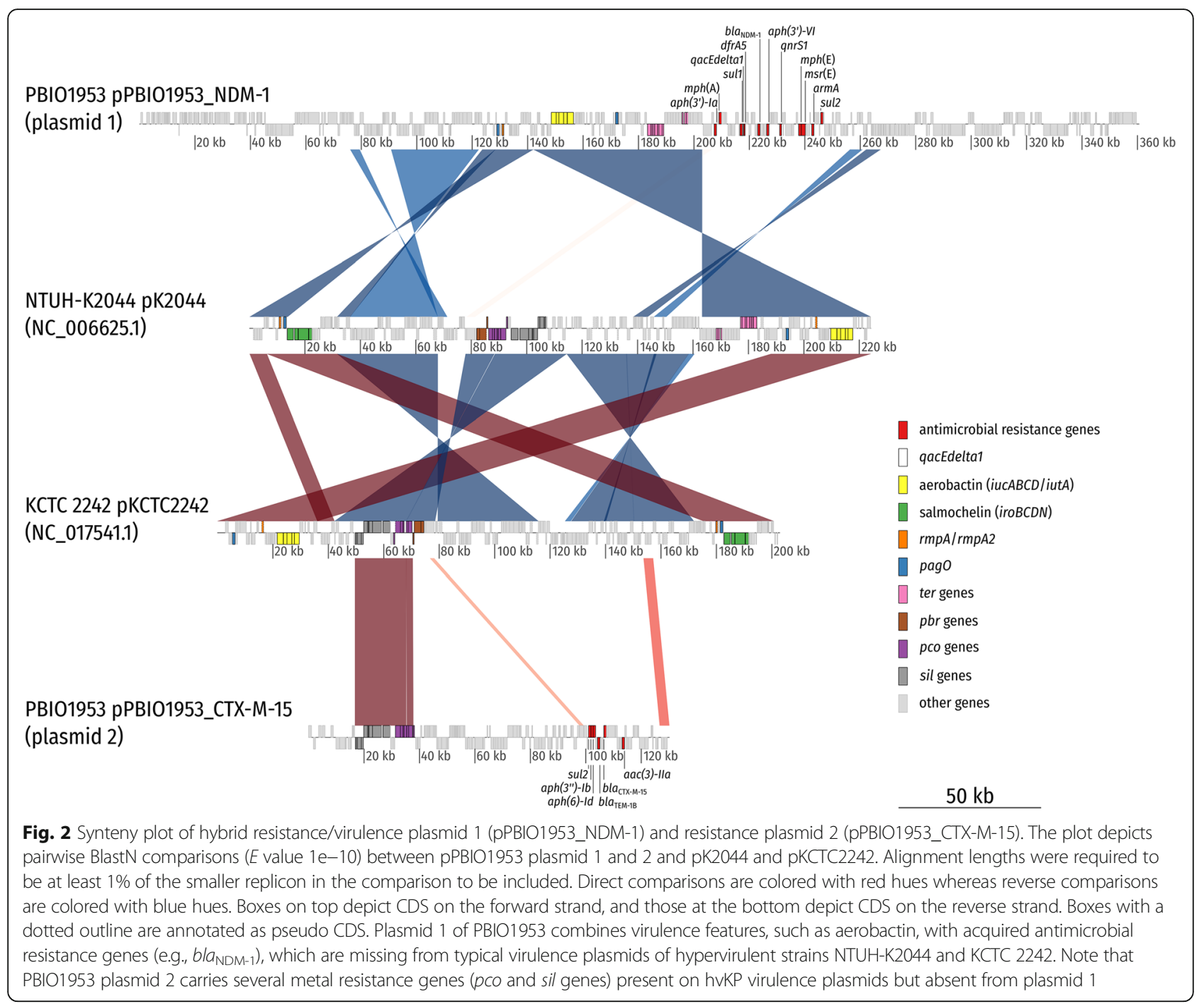

When comparing these plasmid sequences to the other genomes (Fig. 3, Additional file 1: Fig. S2, and Additional file 1: Fig. S3B), we noticed that two non-K. pneumoniae ST307 isolates were also positive for plasmid 1 (NDM-1): (i) PBIO1963 is an E. coli ST362 isolate obtained on September 10, 2019, from patient PT07, who also carried K. pneumoniae ST307 (PBIO1955-obtained on July 24, 2019). We suggest that $K$. pneumoniae ST307 donated plasmid 1 to PBIO1963 within the patient. (ii) PBIO1961 is a K. pneumoniae ST395 isolate. We did not obtain any additional carbapenem-resistant isolates from this patient (PT12), which indicates interpatient transfer of resistance plasmids (Fig. 3); however, our epidemiologic data do not support this suggestion as no other ST307-positive patient was simultaneously present on the same ward. Plasmids 2 and 4 were also present in some non-ST307 genomes. K. pneumoniae ST405 (PBIO1979) isolated on September 25, 2019, from patient PT21 carried plasmid 2 (CTX-M-15). From the same patient, we isolated $K$. pneumoniae ST307 (PBIO1936) on September 18, 2019, again suggesting plasmid intra-bacterial species transfer within this patient. Plasmid 4 (OXA-48) was present in K. pneumoniae ST147 (PBIO1999-isolated from patient PT16 on October 2, 2019) and a K. pneumoniae ST307 isolate (PBIO2000) obtained from the same patient on September 4, 2019. Plasmid 4 was further carried by PBIO1966, a C. freundii ST153 isolate obtained on September 20, 2019, from patient PT02, who additionally carried two $K$. pneumoniae ST307 isolates (PBIO1958: August 5, 2019; PBIO1932: September 14, 2019). It was also present in E. cloacae ST45 (PBIO2014: December 24, 2019), which was the only sequenced isolate from patient PT20. Again, these are examples for putative inter-bacterial plasmid transfers within a patient and among patients, respectively (Fig. 3). Inter-patient transfer for PT20 is further supported by the epidemiologic data: PBIO2012 (ST307) was isolated on December 


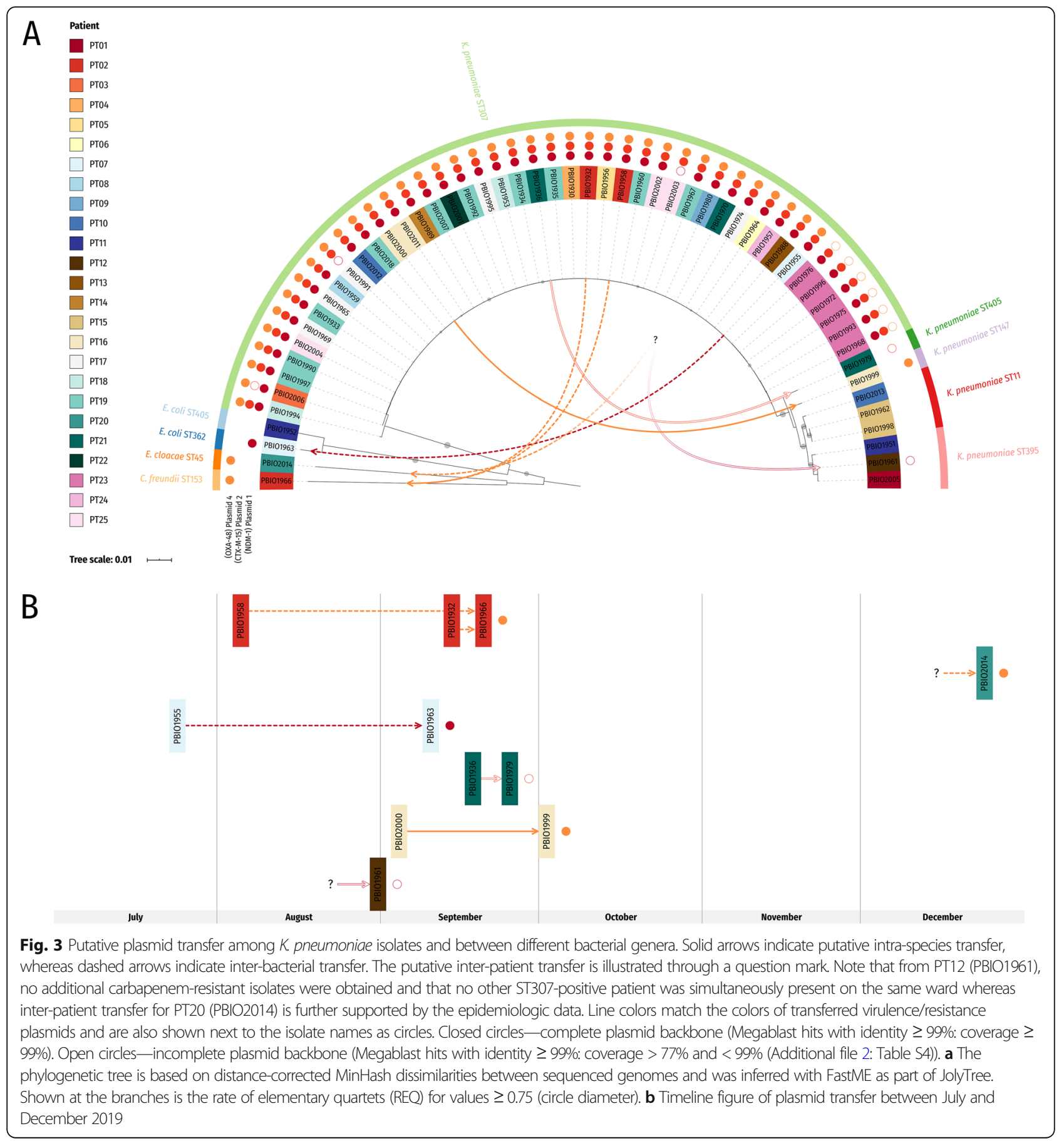

16, 2019, from a putative donor patient (PT10), who stayed on the same ward as PT20.

\section{Phenotypic experiments}

We then investigated the hypothesis that $K$. pneumoniae ST307 outbreak isolates combined high-level antibiotic resistance with fitness, resilience, and virulence features. In several phenotypic experiments, which were selected based on their relevance in the clinical setting and on the presence of resistance and virulence genes in ST307, we investigated five $K$. pneumoniae ST307 isolates from different time points during the outbreak and different patients and hospitals (outbreak isolates), two K. pneumoniae ST395, and one K. pneumoniae ST405 (subsequently termed as the internal group). These were compared to four external K. pneumoniae isolates with 
ST498, ST15, ST307, and ST86 including an archetypal, hypervirulent $K$. pneumoniae strain (hvKP1), which are unrelated to the outbreak. In addition, we included a partially plasmid-cured variant (PCV1935) and controls for each phenotypic experiment.

We observed no significant decreased growth rates $(p>0.1$ at $1,2,3,4$, and $5 \mathrm{~h}$ ) of the ST307 isolates when compared to the internal and external groups and to the E. coli $\mathrm{K}-12$ control (Fig. 4a), and also among each other. PCV1935, which carried the incomplete NDM-1 plasmid 1 , also showed comparable growth $\left(\mu_{1 \mathrm{~h}} \mathrm{ST} 307,1.05\right.$, vs. $\mu_{1 \mathrm{~h}} \mathrm{PCV} 1935,0.67 ; p=0.9999$ ). Interestingly, K. pneumoniae ST395 (PBIO1961) demonstrated a prolonged lag phase $\left(\mu_{1 \mathrm{~h}}\right.$ ST307, 1.05 , vs. $\mu_{1 \mathrm{~h}}$ PBIO1961, $\left.0.51 ; p=0.038\right)$, which will be further explored in a prospective study.

Hypermucoviscosity experiments revealed that all $K$. pneumoniae ST307 outbreak isolates showed strong hypermucoviscosity $(\geq 5 \mathrm{~mm})$ (Fig. 5a), whereas internal K. pneumoniae ST395 and ST405 isolates did not $(p<0.0001)$. Also, the external isolates showed a negative hypermucoid phenotype, with the exception of $K$. pneumoniae ST15 and, unsurprisingly, hvKP1. Hypermucoviscosity of PCV1935 was not significantly different from wildtype PBIO1935, which is interesting given that both plasmid-encoded rmpA and rmpA2 were lost during the curing process. On the contrary, PBIO1961, which carried plasmid 1 (Figs. 3, 4, and 5, and Additional file 1: Figure S3B and S7B), did not show hypermucoviscosity. This genome demonstrated a different rmpA2-truncation and $\mathrm{K}$ locus in comparison to the ST307 clone (Additional file 2: Table S1). Also consider that the different phenotypes might be due to synergy-dependent processes such that plasmids have reduced impacts on other genetic backgrounds than ST307. The hypermucoid phenotype appears to be a fine-tuned process. A recent study [68] showed that loss of $\operatorname{rmpC}$, which is a newly identified gene that contributes to capsule regulation in hvKp, resulted in decreased capsule gene expression while simultaneously retaining hypermucoviscosity. Additional investigations will have to address further which regulatory mechanisms contribute to the hypermucoid phenotype in our outbreak clone. Hypermucoviscosity is associated with invasive and other aggressive types of infection, but recent literature suggests that this characteristic alone is not per se responsible for a hypervirulent phenotype in Klebsiella spp. and that both terms should not be used synonymously $[5,69]$.

Similar to the hypermucoviscosity experiments, we observed significant differences between the K. pneumoniae ST307 outbreak isolates and the internal and external groups regarding their siderophore secretion capacities $(p<0.0001)$ (Fig. 5b, c). They all exhibited a significant higher secretion with average bleaching zone diameters of $20 \mathrm{~mm}$, compared to $8.4 \mathrm{~mm}$ of internal and $10.8 \mathrm{~mm}$ of external isolates (positive control, 12 $\mathrm{mm}$ ). This is likely due to the presence of the NDM-1plasmid-encoded aerobactin, underlined by the results shown for PCV1935, which demonstrated significantly reduced siderophore secretion $(p=0.0025)$ (Fig. 5b, c). Note that hvKP1 also showed increased siderophore secretion compared to the internal group $(p=0.0126)$ but in tendency less than the ST307 outbreak isolates, although this difference was not significant $(p=0.35)$. PBIO1961 registered within the result range of internal and external isolates. This is likely due to a missense mutation of plasmid-encoded iutA and/or the different character of yersiniabactin ("unknown" ybt-Additional file 2: Table S1). Given that hypermucoviscosity, aerobactin secretion, and the metabolite transporter PEG344 [70] are suggested key virulence features of hypervirulent Klebsiella strains during infection, the aboveaverage performance, in addition to extensive antibiotic resistance expression, provides one step closer to explaining why this outbreak evolved. PBIO2009 (external ST307 isolate), which does not possess typical hvKpassociated features like peg-344, iucA, and rmpA [11], showed negative hypermucoid and iron uptake phenotypes (Fig. $5 \mathrm{a}-\mathrm{C}$ ), possibly strengthening our assumption.

We were then interested in whether the K. pneumoniae ST307 outbreak isolates also showed sufficient capacity for survival and resilience in the clinical setting and host. Serum survival experiments revealed high survival rates for all isolates, and together with similar strong biofilm formation capacities (Fig. 4b, c), this suggests that the outbreak $K$. pneumoniae ST307 isolates had good abilities to resist clinical challenges. Comparable results were also obtained for desiccation resilience (Fig. 4d). Despite that internal and external groups showed a tendency to survive 6 days of desiccation at higher rates than $K$. pneumoniae ST307, the difference was not significant after applying Bonferroni correction (outbreak vs. internal group: $p=0.5488$; outbreak vs. external group: $p=0.0730$; internal vs. external group: $p>0.9999$ ).

The genetic characterization revealed several heavy metal efflux genes on plasmid 2. Heavy metal compounds, such as zinc oxide or copper sulfate, are regularly used as feed supplements in livestock, e.g., for prevention of gastro-intestinal disorders and growth promotion in piglets [71], and co-selection of heavy metal and antimicrobial resistance has been increasingly reported $[72,73]$. We thus investigated the bacteria's tolerance by determining minimum inhibitory concentrations (MICs) to copper, zinc, and silver (Additional file 2: Table S1). A MIC value of $1024 \mu \mathrm{g} /$ $\mathrm{mL}$ for copper sulfate was obtained for almost all $K$. 

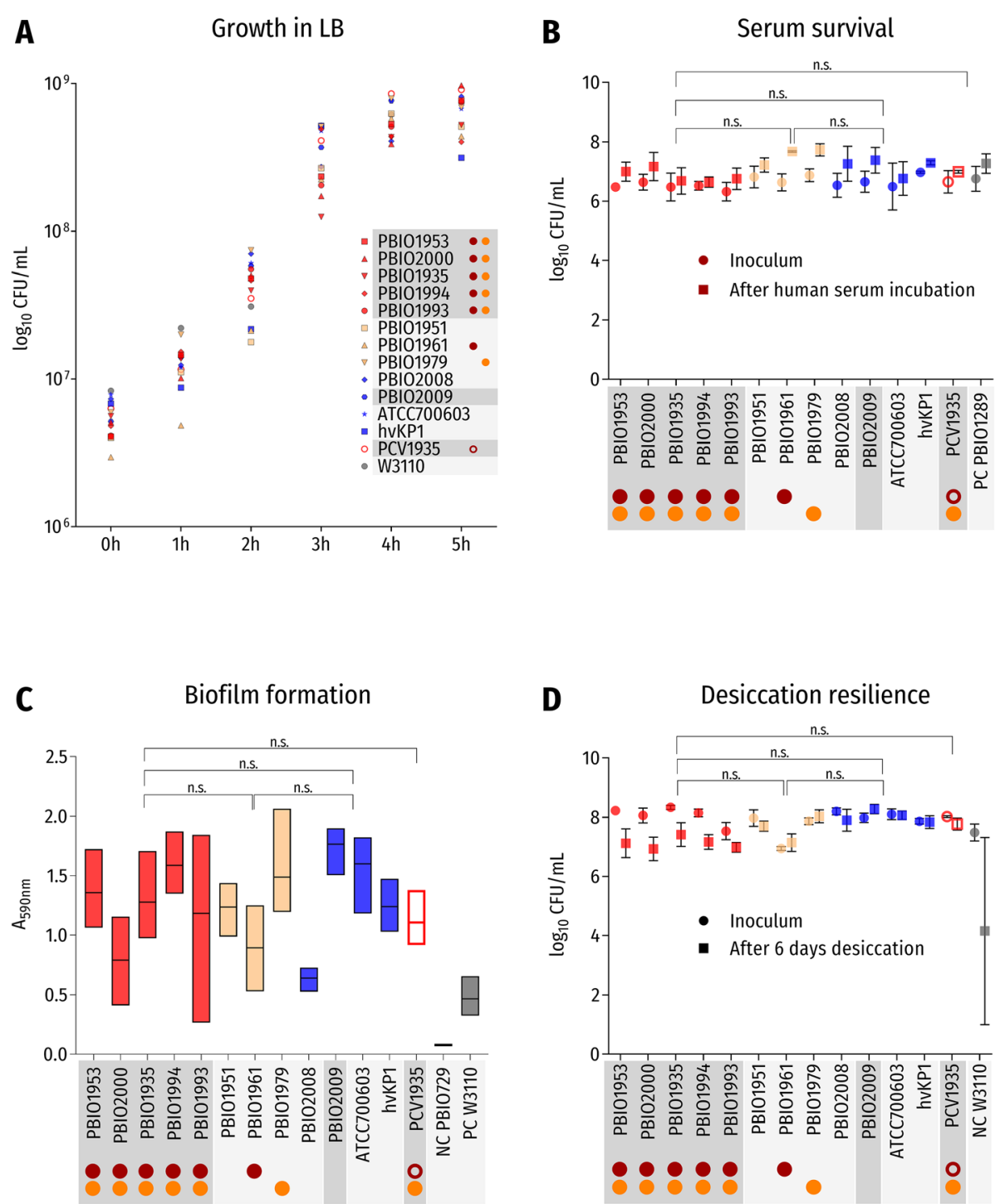

Fig. 4 Results of phenotypic experiments without significant differences. Red symbols: K. pneumoniae ST307 outbreak isolates; yellow symbols: K. pneumoniae non-ST307 internal isolates; blue symbols: K. pneumoniae external isolates. Red circle: occurrence of plasmid 1 in K. pneumoniae ST307 outbreak isolates and PBIO1961; orange circle: occurrence of plasmid 2 in K. pneumoniae ST307 outbreak isolates, PBIO1979, and PCV1935; red open circle: incomplete plasmid 1 in PCV1935. Isolates belonging to ST307 are highlighted in dark gray, and all other isolates in light gray. a Results of growth experiments in LB medium are given as mean values of CFU/mL for each isolate over 5 h. Abbreviations: n.S., not significant. $\mathbf{b}$ Results of serum survival experiments are given as mean values and standard deviation of CFU/mL for each isolate before (inoculum: circles) and after $4 \mathrm{~h}$ of incubation in human serum (squares). c Results of biofilm formation experiments are given as absorbance values (mean with coefficient of variation) of crystal violet at $590 \mathrm{~nm}$ for each isolate after $3 \mathrm{~h}$ of incubation. $\mathbf{d}$ Results of desiccation tolerance experiments are given as mean values and standard deviation of colony-forming units (CFU) per milliliter (CFU/mL) for each isolate before (inoculum: circles) and after 6 days of desiccation (squares)

pneumoniae isolates, both outbreak, internal, and external group isolates (control ATCC25922, $256 \mu \mathrm{g}$ / $\mathrm{mL}$ ), which is an average copper tolerance for Enterobacteriaceae [74]. Interestingly, hvKP1 showed a reduced MIC for copper sulfate $(256 \mu \mathrm{g} / \mathrm{mL})$. We observed similar results for zinc and silver for all isolates (MIC zinc chloride $512 \mu \mathrm{g} / \mathrm{mL}$, control $256 \mu \mathrm{g} /$ $\mathrm{mL}$; silver nitrate $4 \mu \mathrm{g} / \mathrm{mL}$, control $4 \mu \mathrm{g} / \mathrm{mL}$ ), again with the exception of hvKP1 demonstrating a reduced MIC for zinc $(256 \mu \mathrm{g} / \mathrm{mL})$.

\section{Discussion}

To the best of our knowledge, this is the first outbreak in Germany of a K. pneumoniae ST307 clone that produced both NDM-1 and OXA-48 carbapenemases and showed resistance against colistin. It was first detected at 

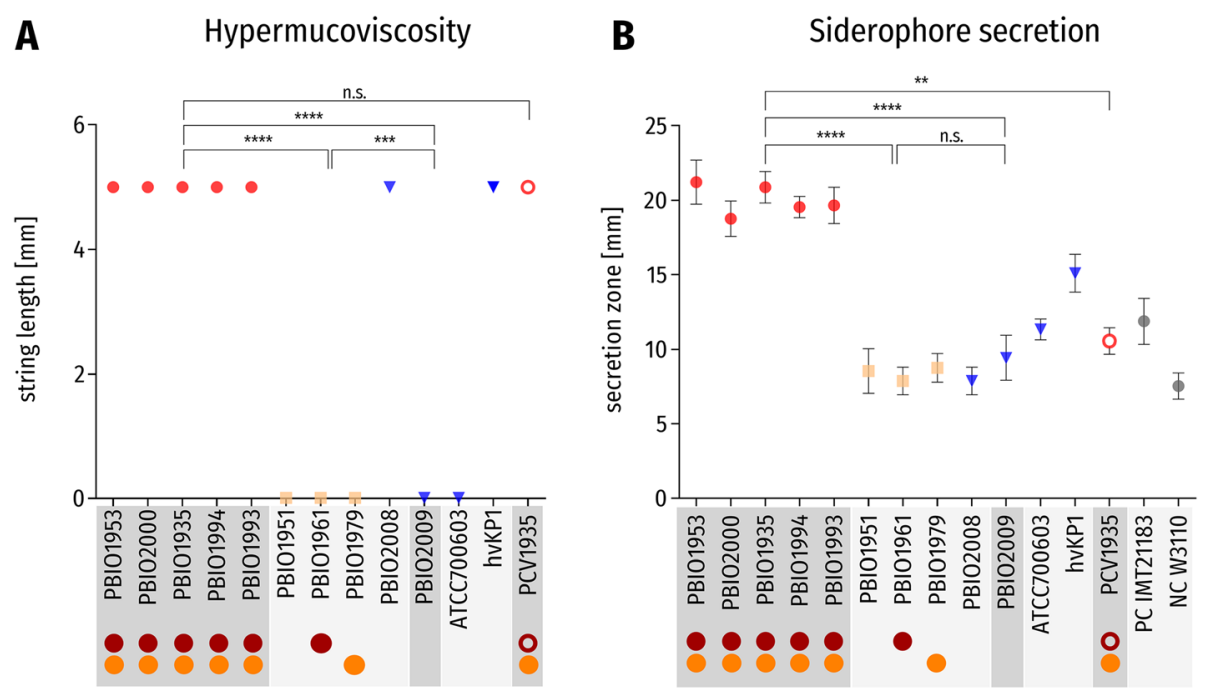

\section{C}

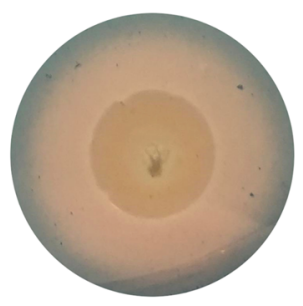

PBI01953

ST307

outbreak isolate

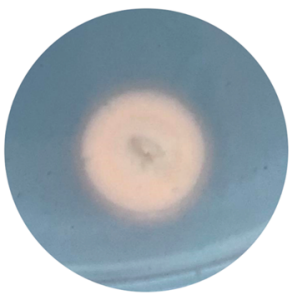

PBI02009

ST307

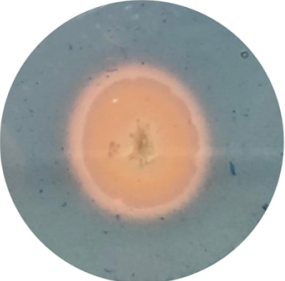

PBI01951

ST395

internal non-ST307 isolate

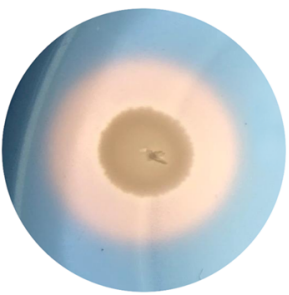

hvKP1

ST86

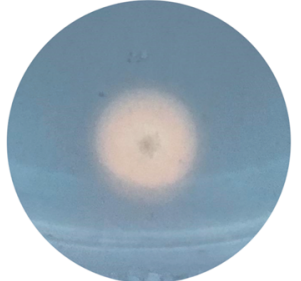

PBI02008

ST15

external non-ST307 isolate

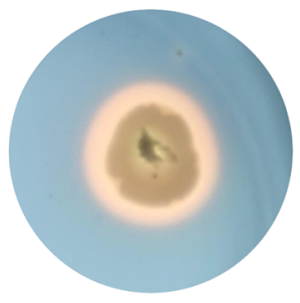

PCV1935

ST307

external isolate

external hypervirulent isolate outbreak plasmid-cured variant

Fig. 5 Results of phenotypic experiments with significant differences. Red symbols: K. pneumoniae ST307 outbreak isolates; yellow symbols: K. pneumoniae nonST307 internal isolates; blue symbols: K. pneumoniae external isolates. Red circle: occurrence of plasmid 1 in K. pneumoniae ST307 outbreak isolates and PBIO1961; orange circle: occurrence of plasmid 2 in K. pneumoniae ST307 outbreak isolates, PBIO1979, and PCV1935; red open circle: incomplete plasmid 1 in PCV1935. Isolates belonging to ST307 are highlighted in dark gray, and all other isolates in light gray. a Results of the hypermucoviscosity test are given as mean values and standard deviation of the string length in millimeters $(\mathrm{mm})$ for each isolate. $\mathbf{b}$ Results of siderophore secretion tests are given as mean values and standard deviation of the secretion zone diameter in millimeters $(\mathrm{mm})$ for each isolate. $\mathbf{c}$ Siderophore secretion experiment on CAS-Agar of six exemplary isolates: PBIO1953 (outbreak isolate [ST307]), PBIO1951 (internal control [ST395]), PBIO2008 (external control [ST15]), PBIO2009 (external control [ST307]), hvKP1 (external hypenirulent control [ST86]), and PCV1935 (outbreak plasmid-cured variant [ST307]). Yellow areas around colonies indicate siderophore secretion. Abbreviations and symbols: n.s., not significant; ${ }^{* * *} p$ value $<0.0001 ;{ }^{* * *} p$ value $<0.001 ;{ }^{* *} p$ value $<0.01$ 
the University Medicine Greifswald in June 2019 from a tracheal secretion sample [19]. As we did not detect any $m c r$-genes, we suggest that colistin resistance is due to chromosomal point mutations including the twocomponent systems PhoP/PhoQ and PmrA/PmrB, which have been previously described in this context [67].

When placing our outbreak clone in a global frame, a cluster of KPC-producing ST307 genomes from the UK was the phylogenetically closest. Interestingly, this cluster was part of a study from 2017 revealing that these genomes harbor genetic features important for clinical and host adaptation, in particular glycogen synthesis [3]. Our outbreak isolates have seemingly developed different resistance phenotypes and virulence strategies, and the UK cluster is thus probably not the true, most likely recent common ancestor of the ST307 outbreak clone.

Although we did not unequivocally verify the hypervirulent character of this clone, it demonstrated hypermucoviscosity, iron uptake, and metabolite transporter capacities-which are relevant for invasive infection and assertiveness in different host environments [41, 69, 70]-comparable to an archetypal hvKp strain [54]. The fact that these key hypervirulence features in addition to disinfectant resistance are found on mutual virulence/resistance plasmids in extensively drug-resistant isolates is concerning and has tremendous public health implications as these mobile genetic elements may be transferred across different bacteria [75]. Our previous work, and those of others, suggests that the combination of high-level drug resistance and virulence is a good combination for the successful spread of bacterial pathogens $[3,53,76-79]$. On the other hand, the co-carriage of plasmid-encoded heavy metal efflux genes did not significantly impact the phenotypic tolerance in the study's K. pneumoniae outbreak isolates, pointing towards that this capacity is less likely a major contributor to the clone's success in this outbreak situation.

We detected identical plasmids among ST307 and other K. pneumoniae isolates as well as other bacterial genera, exacerbating the threat this clone poses across clinical settings. Note, however, that it is possible that ST307 has a greater tolerance towards possible fitness costs of the carried plasmids, implying that donated plasmids might have reduced impacts in other genetic backgrounds [56]. We suggest that the K. pneumoniae ST307 isolates are the general plasmid donors; they were all isolated at an earlier date than the putative acceptor isolates and more independent variants accumulated in some putative acceptor plasmids compared to the $K$. pneumoniae ST307 donor plasmids (Additional file 2: Table S3). The fact that ST395 occurred three times among all isolates but only ST395 PBIO1961 was positive for plasmid 1 additionally strengthens this assumption.
Interestingly, although most hvKp show susceptibility to antimicrobials [44], hvKp with AMR have increasingly emerged in the last decade [80-83], which might be an ongoing trend.

\section{Conclusions}

While the emergence of XDR, virulent, and fit pathogens is worrisome, our study helps to implement control measures and calls for prospective surveillance strategies that take the emergence of "converging" cKp and hvKp pathotypes into account.

\section{Supplementary Information}

The online version contains supplementary material available at https://doi. org/10.1186/s13073-020-00814-6.

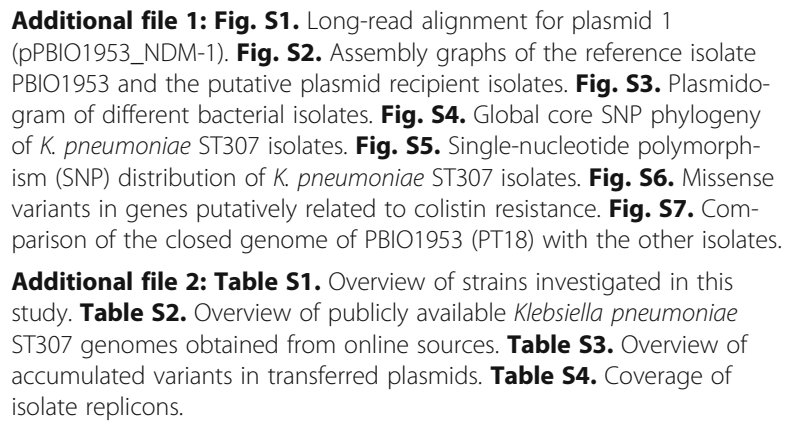

Additional file 2: Table S1. Overview of strains investigated in this study. Table S2. Overview of publicly available Klebsiella pneumoniae ST307 genomes obtained from online sources. Table S3. Overview of accumulated variants in transferred plasmids. Table S4. Coverage of isolate replicons.

\section{Abbreviations}

MDR: Multidrug-resistant; XDR: Extensively drug-resistant; cKp: Classical K. pneumoniae; hvKp: Hypervirulent K. pneumoniae; ST: Sequence type;

MLST: Multi-locus sequence typing; PCV: Plasmid-cured variant; CFU: Colonyforming units; SNP: Single-nucleotide polymorphism; CDS: Coding sequence; UK: United Kingdom

\section{Acknowledgements}

We thank Bettina Rißmann for her excellent technical assistance and Dr. Thomas A. Russo for providing the hvKP1 strain.

\section{Authors' contributions}

K.S. and N.O.H. designed the study. S.E.H., E.E., S.S., SE.G., and J.A.B. performed the phenotypic experiments and the computational analyses. K.S., K.B., A.K., J.A.B., C.D.H., V.B., W.G., S.S., SÖ.G., and T.E. analyzed the data. K.S. and S.E.H. wrote the paper, and all other authors contributed to the writing. All authors read and approved the final version of the manuscript.

\section{Funding}

We acknowledge support for the Article Processing Charge from the DFG (German Research Foundation, 393148499) and the Open Access Publication Fund of the University of Greifswald. The funding sources had no influence on the design of the study and collection, analysis, and interpretation of data, and writing of the manuscript. Open Access funding enabled and organized by Projekt DEAL.

\section{Availability of data and materials}

The experimental and computational data that support the findings of this research are available in this article and its supplementary information files. The genomic data have been deposited in the European Nucleotide Archive (ENA) at EMBL-EBI under accession number PRJEB37933 (https://www.ebi.ac. uk/ena/browser/view/PRJEB37933) [84].

Ethics approval and consent to participate

Ethical approval was given by the ethics committee of the University of Greifswald, Germany (BB 133/20). Informed patient consent was waived as 
samples were taken under a hospital surveillance framework for routine sampling. The research conformed to the principles of the Helsinki Declaration.

\section{Consent for publication}

Not applicable.

\section{Competing interests}

The authors declare that they have no competing interests.

\section{Author details}

${ }^{1}$ Institute of Pharmacy, Pharmaceutical Microbiology, University of Greifswald, Friedrich-Ludwig-Jahn-Str. 17, 17489 Greifswald, Germany. ${ }^{2}$ Central Unit for Infection Prevention and Control, University Medicine Greifswald, Greifswald, Germany. ${ }^{3}$ Friedrich Loeffler-Institute of Medical Microbiology, University Medicine Greifswald, Greifswald, Germany. ${ }^{4}$ Department of General, Visceral, Thoracic and Vascular Surgery, University Medicine Greifswald, Greifswald, Germany. ${ }^{5}$ Institute for Hygiene and Environmental Medicine, University Medicine Greifswald, Greifswald, Germany. ${ }^{6} \mathrm{IMD}$ Laboratory Greifswald, Institute of Medical Diagnostics, Greifswald, Germany. ${ }^{7}$ MVZ Laboratory Limbach Vorpommern-Rügen, Stralsund, Germany. ${ }^{8}$ Department for Infectious Disease Epidemiology, Robert Koch-Institute, Berlin, Germany. ${ }^{9}$ National Reference Centre for Multidrug-Resistant Gram-Negative Bacteria, Ruhr University Bochum, Bochum, Germany. ${ }^{10}$ Institute of Pharmacy, Pharmaceutical Biology, University of Greifswald, Greifswald, Germany.

Received: 2 May 2020 Accepted: 25 November 2020 Published online: 09 December 2020

\section{References}

1. Podschun R, Ullmann U. Klebsiella spp. as nosocomial pathogens: epidemiology, taxonomy, typing methods, and pathogenicity factors. Clin Microbiol Rev. 1998;11(4):589-603.

2. Fazili T, Sharngoe C, Endy T, Kiska D, Javaid W, Polhemus M. Klebsiella pneumoniae liver abscess: an emerging disease. Am J Med Sci. 2016;351(3):297-304.

3. Villa L, Feudi C, Fortini D, Brisse S, Passet V, Bonura C, et al. Diversity, virulence, and antimicrobial resistance of the KPC-producing Klebsiella pneumoniae ST307 clone. Microb Genom. 2017;3(4):e000110.

4. Rossi B, Gasperini ML, Leflon-Guibout V, Gioanni A, de Lastours V, Rossi G, et al. Hypervirulent Klebsiella pneumoniae in cryptogenic liver abscesses, Paris, France. Emerg Infect Dis. 2018;24(2):221-9.

5. Russo TA, Marr CM. Hypervirulent Klebsiella pneumoniae. Clin Microbiol Rev. 2019;32(3):e00001-19.

6. Shon AS, Bajwa RP, Russo TA. Hypervirulent (hypermucoviscous) Klebsiella pneumoniae: a new and dangerous breed. Virulence. 2013;4(2):107-18.

7. Liu C, Guo J. Hypervirulent Klebsiella pneumoniae (hypermucoviscous and aerobactin positive) infection over 6 years in the elderly in China: antimicrobial resistance patterns, molecular epidemiology and risk factor. Ann Clin Microbiol Antimicrob. 2019;18(1):4.

8. Russo TA, Olson R, Fang CT, Stoesser N, Miller M, MacDonald U, et al. Identification of biomarkers for differentiation of hypervirulent Klebsiella pneumoniae from classical K. pneumoniae. J Clin Microbiol. 2018;56(9): e00776-18.

9. Marsh JW, Mustapha MM, Griffith MP, Evans DR, Ezeonwuka C, Pasculle AW, et al. Evolution of outbreak-causing carbapenem-resistant Klebsiella pneumoniae ST258 at a tertiary care hospital over 8 years. Mbio. 2019;10(5):e01945-19.

10. Wyres KL, Hawkey J, Hetland MAK, Fostervold A, Wick RR, Judd LM, et al. Emergence and rapid global dissemination of CTX-M-15-associated Klebsiella pneumoniae strain ST307. J Antimicrob Chemother. 2019;74(3):577-81.

11. Schaufler K, Nowak K, Dux A, Semmler T, Villa L, Kourouma L, et al. Clinically relevant ESBL-producing K. pneumoniae ST307 and E. coli ST38 in an urban West African rat population. Front Microbiol. 2018;9:150.

12. Long SW, Olsen RJ, Eagar TN, Beres SB, Zhao P, Davis JJ, et al. Population genomic analysis of 1,777 extended-spectrum beta-lactamase-producing Klebsiella pneumoniae isolates, Houston, Texas: unexpected abundance of clonal group 307. Mbio. 2017:8(3):e00489-17.

13. Giddins MJ, Macesic N, Annavajhala MK, Stump S, Khan S, McConville TH, et al. Successive emergence of ceftazidime-avibactam resistance through distinct genomic adaptations in blaKPC-2-harboring Klebsiella pneumoniae sequence type 307 isolates. Antimicrob Agents Chemother. 2018;62(3): e02101-17.
14. Novovic K, Trudic A, Brkic S, Vasiljevic Z, Kojic M, Medic D, et al. Molecular epidemiology of colistin-resistant, carbapenemase-producing Klebsiella pneumoniae in Serbia from 2013 to 2016. Antimicrob Agents Chemother. 2017;61(5):e02550-16.

15. Saavedra SY, Diaz L, Wiesner M, Correa A, Arevalo SA, Reyes J, et al. Genomic and molecular characterization of clinical isolates of enterobacteriaceae harboring mcr-1 in Colombia, 2002 to 2016. Antimicrob Agents Ch. 2017;61(12):e00841-17.

16. Baek EH, Kim SE, Kim S, Lee S, Cho OH, In Hong S, et al. Successful control of an extended-spectrum beta-lactamase-producing Klebsiella pneumoniae ST307 outbreak in a neonatal intensive care unit. BMC Infect Dis. 2020;20(1):166.

17. Kim JO, Song SA, Yoon EJ, Shin JH, Lee H, Jeong SH, et al. Outbreak of KPC2-producing Enterobacteriaceae caused by clonal dissemination of Klebsiella pneumoniae ST307 carrying an IncX3-type plasmid harboring a truncated Tn4401a. Diagn Micr Infec Dis. 2017;87(4):343-8.

18. Boonstra MB, Spijkerman DCM, in't Holt AFV, van der Laan RJ, Bode LGM, van Vianen W, et al. An outbreak of ST307 extended-spectrum beta-lactamase (ESBL)producing Klebsiella pneumoniae in a rehabilitation center: an unusual source and route of transmission. Infect Cont Hosp Ep 2020. 41(1):31-36.

19. Haller S, Kramer R, Becker K, Bohnert JA, Eckmanns T, Hans JB, et al. Extensively drug-resistant Klebsiella pneumoniae ST307 outbreak, northeastern Germany, June to October 2019. Euro Surveill. 2019;24(50):1900734.

20. Baym M, Kryazhimskiy S, Lieberman TD, Chung H, Desai MM, Kishony R. Inexpensive multiplexed library preparation for megabase-sized genomes. PLoS One. 2015;10(5):e0128036.

21. Bankevich A, Nurk S, Antipov D, Gurevich AA, Dvorkin M, Kulikov AS, et al. SPAdes: a new genome assembly algorithm and its applications to singlecell sequencing. J Comput Biol. 2012;19(5):455-77.

22. Li H, Durbin R. Fast and accurate short read alignment with BurrowsWheeler transform. Bioinformatics. 2009;25(14):1754-60.

23. Li H, Handsaker B, Wysoker A, Fennell T, Ruan J, Homer N, et al. The Sequence Alignment/Map format and SAMtools. Bioinformatics. 2009;25(16):2078-9.

24. McKenna A, Hanna M, Banks E, Sivachenko A, Cibulskis K, Kernytsky A, et al. The Genome Analysis Toolkit: a MapReduce framework for analyzing nextgeneration DNA sequencing data. Genome Res. 2010;20(9):1297-303.

25. Walker BJ, Abeel T, Shea T, Priest M, Abouelliel A, Sakthikumar S, et al. Pilon: an integrated tool for comprehensive microbial variant detection and genome assembly improvement. PLoS One. 2014;9(11):e112963.

26. Wick RR, Judd LM, Gorrie CL, Holt KE. Unicycler: resolving bacterial genome assemblies from short and long sequencing reads. Plos Comput Biol. 2017; 13(6):e1005595.

27. Li H. Minimap2: pairwise alignment for nucleotide sequences. Bioinformatics. 2018;34(18):3094-100.

28. Milne I, Stephen G, Bayer M, Cock PJ, Pritchard L, Cardle L, et al. Using Tablet for visual exploration of second-generation sequencing data. Brief Bioinform. 2013;14(2):193-202.

29. Wick RR, Schultz MB, Zobel J, Holt KE. Bandage: interactive visualization of de novo genome assemblies. Bioinformatics. 2015;31(20):3350-2.

30. Parks DH, Imelfort M, Skennerton CT, Hugenholtz P, Tyson GW. CheckM: assessing the quality of microbial genomes recovered from isolates, single cells, and metagenomes. Genome Res. 2015;25(7):1043-55.

31. Seemann T. Prokka: rapid prokaryotic genome annotation. Bioinformatics. 2014;30(14):2068-9.

32. Jolley KA, Bray JE, Maiden MCJ. Open-access bacterial population genomics: BIGSdb software, the PubMLST.org website and their applications. Wellcome Open Res. 2018. 3:124.

33. Liu B, Zheng D, Jin Q, Chen L, Yang J. VFDB 2019: a comparative pathogenomic platform with an interactive web interface. Nucleic Acids Res. 2019;47(D1):D687-D92.

34. Zankari E, Hasman H, Cosentino S, Vestergaard M, Rasmussen S, Lund O, et al. Identification of acquired antimicrobial resistance genes. J Antimicrob Chemother. 2012;67(11):2640-4.

35. Carattoli A, Zankari E, Garcia-Fernandez A, Voldby Larsen M, Lund O, Villa L, et al. In silico detection and typing of plasmids using PlasmidFinder and plasmid multilocus sequence typing. Antimicrob Agents Chemother. 2014;58(7):3895-903.

36. Pal C, Bengtsson-Palme J, Rensing C, Kristiansson E, Larsson DGJ. BacMet: antibacterial biocide and metal resistance genes database. Nucleic Acids Res. 2014;42(D1):D737-D43.

37. Alikhan NF, Petty NK, Ben Zakour NL, Beatson SA. BLAST Ring Image Generator (BRIG): simple prokaryote genome comparisons. BMC Genomics. 2011;12:402. 
38. Camacho C, Coulouris G, Avagyan V, Ma N, Papadopoulos J, Bealer K, et al. BLAST+: architecture and applications. BMC Bioinformatics. 2009;10:421.

39. Page AJ, Cummins CA, Hunt M, Wong VK, Reuter S, Holden MT, et al. Roary: rapid large-scale prokaryote pan genome analysis. Bioinformatics. 2015;31(22):3691-3.

40. Lam MMC, Wick RR, Wyres KL, Gorrie CL, Judd LM, Jenney AWJ, et al. Genetic diversity, mobilisation and spread of the yersiniabactin-encoding mobile element ICEKp in Klebsiella pneumoniae populations. Microb Genom. 2018;4(9):e000196.

41. Lam MMC, Wyres KL, Judd LM, Wick RR, Jenney A, Brisse S, et al. Tracking key virulence loci encoding aerobactin and salmochelin siderophore synthesis in Klebsiella pneumoniae. Genome Med. 2018;10(1):77.

42. Wick RR, Heinz E, Holt KE, Wyres KL. Kaptive web: user-friendly capsule and lipopolysaccharide serotype prediction for Klebsiella genomes. J Clin Microbiol. 2018;56(6):e00197-18.

43. Wyres KL, Wick RR, Gorrie C, Jenney A, Follador R, Thomson NR, et al. Identification of Klebsiella capsule synthesis loci from whole genome data. Microb Genom. 2016;2(12):e000102.

44. Wang X, Xie Y, Li G, Liu J, Li X, Tian L, et al. Whole-genome-sequencing characterization of bloodstream infection-causing hypervirulent Klebsiella pneumoniae of capsular serotype K2 and ST374. Virulence. 2018;9(1):510-21.

45. Shin SH, Kim S, Kim JY, Lee S, Um Y, Oh MK, et al. Complete genome sequence of the 2,3-butanediol-producing Klebsiella pneumoniae strain KCTC 2242. J Bacteriol. 2012;194(10):2736-7.

46. Guy L, Kultima JR, Andersson SG. genoPlotR: comparative gene and genome visualization in R. Bioinformatics. 2010;26(18):2334-5.

47. Croucher NJ, Page AJ, Connor TR, Delaney AJ, Keane JA, Bentley SD, et al. Rapid phylogenetic analysis of large samples of recombinant bacterial whole genome sequences using Gubbins. Nucleic Acids Res. 2015;43(3):e15-e.

48. Page AJ, Taylor B, Delaney AJ, Soares J, Seemann T, Keane JA, et al. SNPsites: rapid efficient extraction of SNPs from multi-FASTA alignments. Microb Genom. 2016;2(4):e000056.

49. Kozlov AM, Darriba D, Flouri T, Morel B, Stamatakis A. RAxML-NG: a fast, scalable and user-friendly tool for maximum likelihood phylogenetic inference. Bioinformatics. 2019;35(21):4453-5.

50. Letunic I, Bork P. Interactive Tree Of Life (iTOL) v4: recent updates and new developments. Nucleic Acids Res. 2019;47(W1):W256-W9.

51. Criscuolo A. A fast alignment-free bioinformatics procedure to infer accurate distance-based phylogenetic trees from genome assemblies. Res Ideas Outcomes. 2019;5:e36178.

52. Karp PD, Riley M, Saier M, Paulsen IT, Collado-Vides J, Paley SM, et al. The EcoCyc database. Nucleic Acids Res. 2002;30(1):56-8.

53. Turton J, Davies F, Turton J, Perry C, Payne Z, Pike R. Hybrid resistance and virulence plasmids in "high-risk" clones of Klebsiella pneumoniae, including those carrying blaNDM-5. Microorganisms. 2019;7(9):326.

54. Russo TA, Gill SR. Draft genome sequence of the hypervirulent Klebsiella pneumoniae strain hvKP1, isolated in Buffalo, New York. Genome Announc. 2013;1(2):e0006513.

55. Schaufler K, Wieler LH, Semmler T, Ewers C, Guenther S. ESBL-plasmids carrying toxin-antitoxin systems can be "cured" of wild-type Escherichia coli using a heat technique. Gut Pathog. 2013;5(1):34

56. Schaufler K, Semmler T, Pickard DJ, de Toro M, de la Cruz F, Wieler LH, et al. Carriage of extended-spectrum beta-lactamase-plasmids does not reduce fitness but enhances virulence in some strains of pandemic E coli lineages. Front Microbiol. 2016;7:336.

57. Schwyn B, Neilands JB. Universal chemical-assay for the detection and determination of siderophores. Anal Biochem. 1987;160(1):47-56.

58. Schaufler K, Semmler T, Wieler LH, Trott DJ, Pitout J, Peirano G, et al. Genomic and functional analysis of emerging virulent and multidrugresistant Escherichia coli lineage sequence type 648. Antimicrob Agents Chemother. 2019;63(6):e00243-19.

59. Du XJ, Wang XY, Dong X, Li P, Wang S. Characterization of the desiccation tolerance of Cronobacter sakazakii strains. Front Microbiol. 2018;9:2867.

60. Ranjan A, Scholz J, Semmler T, Wieler LH, Ewers C, Muller S, et al. ESBLplasmid carriage in E coli enhances in vitro bacterial competition fitness and serum resistance in some strains of pandemic sequence types without overall fitness cost. Gut Pathog. 2018;10:24.

61. Schierack P, Heiden SE, Khan MM, Nikolaus L, Kolenda R, Stubbe M, et al. Genomic and phenotypic analysis of an ESBL-producing E. coli ST1159 clonal lineage from wild birds in Mongolia. Front Microbiol. 2020;11:1699.

62. Kruskal WH, Wallis WA. Use of ranks in one-criterion variance analysis. J Am Stat Assoc. 1952;47(260):583-621.
63. Sedgwick P. Multiple hypothesis testing and Bonferroni's correction. BMJ. 2014;349:g6284.

64. Marsh JW, Krauland MG, Nelson JS, Schlackman JL, Brooks AM, Pasculle AW, et al. Genomic epidemiology of an endoscope-associated outbreak of Klebsiella pneumoniae carbapenemase (KPC)-producing K. pneumoniae. Plos One. 2015;10(12):e0144310.

65. Haft RJF, Palacios G, Nguyen T, Mally M, Gachelet EG, Zechner EL, et al. General mutagenesis of $\mathrm{F}$ plasmid Tral reveals its role in conjugative regulation. J Bacteriol. 2006;188(17):6346-53.

66. Marchese A, Debbia EA. The role of gyrA, gyrB, and dnaA functions in bacterial conjugation. Ann Microbiol. 2016;66(1):223-8.

67. Olaitan AO, Morand S, Rolain JM. Mechanisms of polymyxin resistance: acquired and intrinsic resistance in bacteria. Front Microbiol. 2014;5:643.

68. Walker KA, Miner TA, Palacios M, Trzilova D, Frederick DR, Broberg CA, et al. A Klebsiella pneumoniae regulatory mutant has reduced capsule expression but retains hypermucoviscosity. Mbio. 2019;10(2):e00089-19.

69. Catalan-Najera JC, Garza-Ramos U, Barrios-Camacho H. Hypervirulence and hypermucoviscosity: two different but complementary Klebsiella spp. phenotypes? Virulence. 2017;8(7):1111-23.

70. Bulger J, MacDonald U, Olson R, Beanan J, Russo TA. Metabolite transporter PEG344 is required for full virulence of hypervirulent Klebsiella pneumoniae strain hvKP1 after pulmonary but not subcutaneous challenge. Infect Immun. 2017:85(10):e00093-17.

71. Adamse $P, H J v E, A \vee P, B i k k e r P, J d$ J. Trend analysis of copper and zinc in animal feed. Wageningen: Rikilt - Institute of Food Safety; 2011.

72. Baker-Austin C, Wright MS, Stepanauskas R, McArthur JV. Co-selection of antibiotic and metal resistance. Trends Microbiol. 2006;14(4):176-82.

73. van Alen S, Kaspar U, Idelevich EA, Kock R, Becker K. Increase of zinc resistance in German human derived livestock-associated MRSA between 2000 and 2014. Vet Microbiol. 2018;214:7-12.

74. Ghazisaeedi F, Ciesinski L, Bednorz C, Johanns V, Pieper R, Tedin K, et al. Phenotypic zinc resistance does not correlate with antimicrobial multiresistance in fecal E. coli isolates of piglets. Gut Pathog. 2020. 12(4).

75. Evans DR, Griffith MP, Sundermann AJ, Shutt KA, Saul MI, Mustapha MM, et al. Systematic detection of horizontal gene transfer across genera among multidrug-resistant bacteria in a single hospital. Elife. 2020;9:e53886.

76. Ewers C, Bethe A, Stamm I, Grobbel M, Kopp PA, Guerra B, et al. CTX-M-15D-ST648 Escherichia coli from companion animals and horses: another pandemic clone combining multiresistance and extraintestinal virulence? J Antimicrob Chemother. 2014;69(5):1224-30.

77. Beceiro A, Tomas M, Bou G. Antimicrobial resistance and virulence: a successful or deleterious association in the bacterial world? Clin Microbiol Rev. 2013;26(2):185-230.

78. Calhau V, Ribeiro G, Mendonca N, Da Silva GJ. Prevalent combination of virulence and plasmidic-encoded resistance in ST 131 Escherichia coli strains. Virulence. 2013;4(8):726-9.

79. Pitout JD. Extraintestinal pathogenic Escherichia coli: a combination of virulence with antibiotic resistance. Front Microbiol. 2012;3:9.

80. Surgers L, Boyd A, Girard PM, Arlet G, Decre D. ESBL-producing strain of hypervirulent Klebsiella pneumoniae K2. France Emerg Infect Dis. 2016;22(9):1687-8.

81. Zhang Y, Zeng J, Liu W, Zhao F, Hu Z, Zhao C, et al. Emergence of a hypervirulent carbapenem-resistant Klebsiella pneumoniae isolate from clinical infections in China. J Inf Secur. 2015;71(5):553-60.

82. Shankar C, Nabarro LE, Devanga Ragupathi NK, Muthuirulandi Sethuvel DP, Daniel JL, Doss CG, et al. Draft genome sequences of three hypervirulent carbapenem-resistant Klebsiella pneumoniae isolates from bacteremia. Genome Announc. 2016;4(6):e01081-16.

83. Gu D, Dong N, Zheng Z, Lin D, Huang M, Wang L, et al. A fatal outbreak of ST11 carbapenem-resistant hypervirulent Klebsiella pneumoniae in a Chinese hospital: a molecular epidemiological study. Lancet Infect Dis. 2018;18(1):37-46.

84. Heiden SE, Hübner NO, Bohnert JA, Heidecke CD, Kramer A, Balau V, et al. A Klebsiella pneumoniae ST307 outbreak clone from Germany demonstrates features of extensive drug resistance, hypermucoviscosity and enhanced iron acquisition. Eur Nucleotide Arch. 2020. https://www.ebi.ac.uk/ena/ browser/view/PRJEB37933.

\section{Publisher's Note}

Springer Nature remains neutral with regard to jurisdictional claims in published maps and institutional affiliations. 